\title{
Der Migrationshintergrund in multikulturellen Pflegearrangements
}

\section{Ergebnisse einer qualitativen und quantitativen Befragung älterer Türkeistämmiger}

\author{
Verena Krobisch¹ (Dipl. Soz), Pia-Theresa Sonntag' (Dipl. Soz), \\ Kübra Gül' (M.A. Erziehungswissenschaftlerin), Polina Aronson² (PhD), \\ Liane Schenk ${ }^{1}$ (Dr. phil.)
}

${ }^{1}$ Institut für Medizinische Soziologie und Rehabilitationswissenschaft, Charité - Universitätsmedizin Berlin

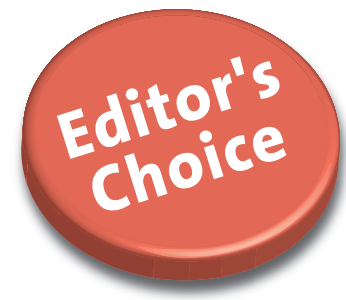

${ }^{2}$ Deutsches Institut für Menschenrechte, Berlin

\begin{abstract}
Zusammenfassung:
Hintergrund: Migration befördert die Zunahme multikultureller Pflegearrangements. Welche Akzeptanz diese Versorgungsbeziehungen auf Nutzerseite finden, ist kaum erforscht.

Ziel: Untersucht wird, ob und inwiefern für ältere Türkeistämmige ein gemeinsamer Migrations- bzw. soziokultureller Hintergrund mit den Pflegefachpersonen relevant ist.

Methode: Im Sinne einer Datentriangulation wurden Ergebnisse einer qualitativen und quantitativen Studie zu Pflegeerwartungen älterer Türkeistämmiger zusammengeführt. Die Datengrundlage bilden leitfadengestützte Interviews und FragebogenInterviews. Die Auswertung erfolgte mittels dokumentarischer Methode nach Bohnsack sowie deskriptiver und multivariater Analysen.

Ergebnisse: Eine Berücksichtigung kulturell- und migrationsbedingter Aspekte in der Pflege ist für die Mehrheit der Befragten bedeutsam. Mehr als drei Viertel erachten Türkischkenntnisse der Pflegefachpersonen als ein Merkmal guter Pflege. Dabei kommt es innen auf die Möglichkeit zur sachlichen, aber auch zur kulturell geprägten intuitiven Kommunikation in der Muttersprache an. Ein geringer Grad an Deutschkenntnissen, eine türkisch geprägte Identität sowie das weibliche Geschlecht sind mit einem Bedarf an migrationssensibler Pflege assoziiert.

Schlussfolgerungen: Ein gemeinsamer soziokultureller Hintergrund mit den Pflegefachpersonen und die gemeinsame Muttersprache scheinen für ältere Türkeistämmige eine Voraussetzung für gute Pflege. Weitere Forschung sollte klären, unter welchen Bedingungen multikulturelle Pflegekonstellationen in dieser Gruppe auf Akzeptanz stoßen.
\end{abstract}

Schlüsselwörter: Multikulturelle Pflegearrangements, Pflegeerwartungen, türkeistämmige Migrantlnnen

The migration background in multicultural care settings - results of a qualitative and quantitative survey of elderly migrants from Turkey

\section{Abstract:}

Background: Migration is associated with an increase of multicultural care settings. The acceptance of such care relations from user's point of view has been rarely explored yet.

Aim: It is examined, if and how elderly migrants from Turkey consider a common migration background respectively a common socio-cultural background of caregivers as relevant.

Method: In terms of data triangulation results of a qualitative study and a quantitative study on care expectations of elderly migrants from Turkey have been merged. Data was collected by means of guideline-based and standardised interviews. Analysis included the documentary method according to Bohnsack as well as descriptive and multivariate methods.

Results: Cultural and migration-related aspects are considered relevant by the vast majority of respondents. Turkish language skills of caregivers are important to more than three-quarters. According to qualitative results, the possibility to objective as well as culturally shaped intuitive communication in the mother tongue is crucial. Correspondingly, a low level of German language skills and a Turkish ethnic identity are associated with a need of migration-sensitive care.

Conclusions: A common socio-cultural background with caregivers and the common mother tongue appear to be prerequisites of good care for elderly migrants from Turkey. Further research should examine the conditions under which multicultural care settings are accepted in this group.

Keywords: multicultural care settings, care expectations, migrants from Turkey 
Was ist (zu dieser Thematik) schon bekannt?

Erkenntnisse zur Akzeptanz multikultureller Versorgungsbeziehungen in der professionellen Pflege aus Nutzerperspektive sind kaum vorhanden

Was ist neu?

Ältere Türkeistämmige messen einem gemeinsamen Migrationsbzw. soziokulturellen Hintergrund mit den Pflegenden hohe Relevanz bei.

Welche Konsequenzen haben die Ergebnisse für die Pflegepraxis? Pflegerische Versorgung sollte dem Bedürfnis Rechnung tragen, sich in der Muttersprache zu verständigen und auf einer intuitiven Ebene verstanden zu werden.

\section{Einleitung}

Prognosen berechnen einen weiterhin zunehmenden Fachkräftemangel auf dem Pflegemarkt in Deutschland bei gleichzeitig wachsenden Zahlen an Pflegebedürftigen (vgl. Afentakis \& Maier, 2010; Braeseke et al., 2013). Zur Reduktion des Fachkräftemangels in der professionellen Pflege wurden vielfältige Maßnahmen initiiert, um die Attraktivität des Pflegeberufs zu erhöhen (vgl. Bundesministerium für Gesundheit, 2015). Die Maßnahmen zielen sowohl darauf $\mathrm{ab}$, mehr Menschen aus der inländischen Bevölkerung - einschließlich jener mit Migrationshintergrund -, als auch Pflegefachpersonen und Auszubildende aus dem Ausland für eine Tätigkeit in der Pflege zu gewinnen. Folglich wächst in diesem Berufsfeld die Zahl der Fachkräfte mit Migrationshintergrund.

Zugleich nimmt die Zahl der Pflegebedürftigen mit Migrationshintergrund zu. Die Bevölkerung mit Migrationshintergrund über 65 Jahre zählt zu den am schnellsten wachsenden Bevölkerungsgruppen in Deutschland. Prognostiziert wird ein Anstieg von derzeit 1,4 Millionen bis zum Jahr 2032 auf 3,6 Millionen (vgl. Schimany, Rühl \& Kohls, 2012). Studien weisen nachdrücklich darauf hin, dass bei älteren MigrantInnen in den nächsten Jahren mit einem erhöhten Pflegebedarf gerechnet werden muss, was einerseits durch ihren zahlenmäßigen Zuwachs und andererseits durch ihren Gesundheitszustand bedingt ist (vgl. Bundesministerium für Gesundheit, 2011; Kohls, 2011, 2012). Eine Herausforderung der pflegerischen Versorgung wird es daher sein, den sozioökonomischen, soziokulturellen und sprachlichen Besonderheiten von MigrantInnen Rechnung zu tragen.

Mit der Zunahme von Pflegefachpersonen sowie Pflegebedürftigen mit Migrationshintergrund geht eine ethnische und kulturelle Pluralisierung in der Pflege einher. Die hieraus resultierenden multikulturellen Pflegearrangements setzen sich aus zugewanderten Pflegefachpersonen sowie Pflegenden ohne Migrationshintergrund zusammen, welche ältere Menschen ohne Migrationshintergrund, aber auch mit einem anderen als dem eigenen Migrationshintergrund versorgen. Trotz des Bedeutungszuwachses multikultureller Pflegearrangements ist bislang wenig bekannt zur Akzeptanz und zu den Effekten solcher Konstellationen. Untersucht wurden Dynamiken innerhalb «multikultureller Teams» und deren
Einfluss auf die Pflegequalität (vgl. Stagge, 2016). Jedoch fehlt es an einer systematischen Auseinandersetzung mit multikulturellen Pflegekonstellationen, in der die Beziehung zwischen Pflegenden und Pflegeempfängern im Fokus steht.

Türkeistämmige - aus der Türkei Zugewanderte unterschiedlicher ethnischer Zugehörigkeit - bilden mit einem Anteil von 11,9\% die derzeit größte Gruppe der rund 1,5 Mio. Menschen mit Migrationshintergrund im Alter von 65 Jahren oder mehr in Deutschland (vgl. Schimany, Rühl \& Kohls, 2012). Aufgrund ihrer häufig prekären Arbeitsund Lebensbedingungen ist bei dieser Migrantengruppe auch von einem erhöhten Pflegebedarf auszugehen (vgl. z.B. Dietzel-Papakyriaku \& Olbermann, 2005; Özcan \& Seifert, 2006; Schenk, 2008; Wengler, 2013). Wenngleich der Pflege durch Angehörige in der türkeistämmigen Bevölkerung nach wie vor ein hoher Stellenwert beigemessen wird (vgl. Strumpen 2012; Kohls 2012), lösen sich auch hier familiale Strukturen auf und andere Versorgungsformen, insbesondere ambulante professionelle Pflege, gewinnen an Bedeutung. Inwieweit sich multikulturelle Pflegekonstellationen für die Versorgung älterer Türkeistämmiger eignen, wurde bislang kaum erforscht. Ergebnisse zu den Alters- und Pflegevorstellungen sowie zur Inanspruchnahme professioneller Pflege deuten lediglich darauf hin, dass soziokulturelle Differenzen bei der Pflege älterer Türkeistämmiger eine Rolle spielen. So wird eine geringe Inanspruchnahme professioneller Gesundheitsund Pflegeleistungen durch ältere Türkeistämmige auf kulturelle und sprachliche Barrieren sowie fehlende kultur- und migrationssensible Pflegeangebote zurückgeführt (vgl. z.B. Okken, Spallek \& Razum, 2008; Ulusoy \& Gräßel, 2010). Die Annahme Türkeistämmiger, das Gesundheitspersonal in Deutschland wüsste zu wenig über ihre Kultur, gilt hierbei als ein zentrales Hemmnis der Inanspruchnahme (vgl. Bermejo, Hölzel, Kriston \& Härter, 2012). Ergebnisse, die gesicherte Rückschlüsse auf die Akzeptanz von Pflegepersonen mit einem anderen Migrations- bzw. soziokulturellen Hintergrund und somit auf die Tragfähigkeit multikultureller Pflegekonstellationen für diese Gruppe zulassen, sind nicht vorhanden.

\section{Zielsetzung und Fragestellung}

Ziel des Beitrags ist es, den Blick auf die Tragfähigkeit multikultureller Pflegearrangements aus Nutzerperspektive zu lenken. Zentral ist hier die Frage, welche Relevanz ältere türkeistämmige MigrantInnen einem gemeinsamen Migrations- bzw. soziokulturellen Hintergrund mit Pflegefachpersonen beimessen und inwieweit eine migrationssensible Angebotsausrichtung bedeutsam ist. Mit Beantwortung dieser Frage für in Berlin lebende ältere Türkeistämmige wird ein Erkenntnisbeitrag geleistet, der für multikulturelle Pflegekonstellationen und die Gestaltung professioneller Pflege allgemein von Interesse sein kann. 


\section{Methode}

Datengrundlage bilden eine qualitative Studie (Schenk, Meyer, Maier, Aronson \& Gül, 2011) und eine darauf aufbauende quantitativ ausgerichtete Studie (Schenk, Krobisch \& Ikiz, 2014) zu Alterns- und Pflegeorientierungen älterer MigrantInnen. Beide Studien folgen einem induktiven Ansatz. Die qualitativ angelegte Studie diente zunächst der Rekonstruktion zentraler Orientierungsmuster, während die darauf aufbauende Studie diese quantifizieren und in einen statistischen Zusammenhang zu weiteren relevanten Parametern stellen sollte. Für den vorliegenden Beitrag wurden im Sinne einer Datentriangulation ausgewählte Ergebnisse beider Studien erstmals zusammengeführt und durch bislang nicht ausgewertete Aspekte sowie eine multivariate Analyse ergänzt.

\section{Qualitative Studie}

Die qualitative Studie schloss MigrantInnen zweier Altersgruppen ein (60 Jahre und älter sowie zwischen 45 und 55 Jahren), die aus der Türkei und aus dem postsowjetischen Raum stammten. Zwischen November 2010 und Mai 2011 wurden insgesamt 48 leitfadengestützte narrative Interviews realisiert, davon elf mit türkeistämmigen ArbeitsmigrantInnen der ersten Einwanderergeneration. Diese stehen im Zentrum der folgenden Ergebnisdarstellung. Mit leitfadengestützten narrativen Interviews und der Auswertung nach der Dokumentarischen Methode wurde ein qualitatives rekonstruktives Verfahren realisiert. Damit wurde dem Umstand Rechnung getragen, dass den AkteurInnen ihre alterns- und pflegebezogenen Orientierungen nicht bewusst sein müssen, sondern diese im Bereich des unreflektierten und sogenannten «atheoretischen» Wissens liegen können. Solche impliziten, aber handlungsleitenden Orientierungen dokumentieren sich in der Handlungspraxis von Individuen. Ein Zugang zu dieser Handlungspraxis sind Alltagserzählungen, die mittels narrativer Leitfadeninterviews erhoben und anhand biografieorientierter Erzählstimuli angestoßen werden können (Tabelle 1). Mit einer qualitativen Herangehensweise sollte zudem vermieden werden, das bis dato relativ wenig erkundete Forschungsfeld mit theoretischen Vorannahmen vorzustrukturieren, wie es etwa mit einem hypothesengeleiteten Vorgehen der Fall ist. Die biografischen Erzählstimuli sorgten weiterhin dafür, dass die Befragten das Interview zunächst selbst strukturierten, indem sie eine Auswahl an relevanten Themen aus ihrer Lebenswelt trafen und diese «ungestört» erzählen konnten. Dadurch wurde eine Selbstläufigkeit des Interviews gewährleistet. Diese Selbstläufigkeit ist aus methodischer Sicht vonnöten, damit die Interviewten ihre sogenannten «Relevanzsysteme» (Bohnsack, 2008) entfalten und so die «relevanten» Lebensthemen bzw. Orientierungen methodisch zugänglich werden können. Ergänzt wurden die standardisiert zu stellenden Eingangsfragen durch flexibel handzuhabende themenzentrierte Fragen, die Er-
Tabelle 1. Auszug aus dem Interviewleitfaden

\begin{tabular}{lll}
\hline Phase & Eingangsfragen aus dem Interviewleitfaden \\
\hline $\begin{array}{l}\text { Biografischer } \\
\text { Erzählstimulus }\end{array}$ & 1. & $\begin{array}{l}\text { Ich möchte gern, dass Sie mir } \\
\text { zunächst von Ihrem Leben erzählen. }\end{array}$ \\
& 1.1 & $\begin{array}{l}\text { Und bevor Sie nach Deutschland } \\
\text { kamen? Erzählen Sie mir bitte } \\
\text { von Ihrem Leben, bevor Sie nach } \\
\text { Deutschland kamen. } \\
\end{array}$ \\
& 1.2 & $\begin{array}{l}\text { Und heute? Erzählen Sie von Ihrem } \\
\text { Leben heute. }\end{array}$ \\
\hline
\end{tabular}

Anmerkung. 1.1. und 1.2. wurden erst dann explizit eingeführt, wenn sich die Interviewten zu diesen Themen nicht geäußert hatten.

zählungen in den Themenfeldern «Alter» und «Pflege» anstoßen sollten.

Migrationsspezifische Teilnahmebarrieren an Gesundheitsstudien, von denen die Sprachbarriere nur eine ist, sind vielfach diskutiert und belegt (vgl. Borde, 2005; Razum \& Zeeb, 1998; Reiss et al. 2014; Sass et al., 2015; Schenk, 2002). Für Studien im sensiblen Feld der Pflege, welches mit Privatheit, Intimsphäre und zahlreichen tabubesetzten Themen wie Pflegebedürftigkeit, Tod und Sterben assoziiert ist, werden zusätzliche Zugangsbarrieren konstatiert (vgl. Yilmaz, Glodny \& Razum, 2009). Die InterviewteilnehmerInnen wurden daher mit Hilfe eines Netzwerkansatzes gewonnen, der eine Einbindung von in der jeweiligen ethnischen Community verankerten Vertrauenspersonen in die Teilnehmergewinnung vorsieht und sich am Konzept der «Sozialen Netzwerkarbeit» von Yilmaz et al. (2009) orientiert. Als Vertrauenspersonen einbezogen wurden VertreterInnen von Migrantenverbänden, Sprachvereinen, Kulturzentren, dem internationalen Pflegehaus Kreuzberg in Berlin, Moscheen und ähnlichen Einrichtungen. Dabei wurde eine theoretische SamplingStrategie verfolgt, die eine Einbeziehung für die Fragestellung relevanter Fälle gewährleisten sollte. Durch eine kriteriengesteuerte Teilnehmergewinnung wurde versucht, theoretisch relevante Merkmalskombinationen möglichst umfassend zu berücksichtigen (vgl. Kelle \& Kluge, 1999). Solche Merkmale waren u.a. Alter, Geschlecht, Bildung, Familienstand sowie Herkunftsland und Zuwanderungskontext. Die Interviews dauerten zwischen 30 und $120 \mathrm{Mi}$ nuten bei einer durchschnittlichen Interviewdauer von 67 Minuten. Die zumeist in türkischer Sprache geführten Interviews wurden tonbandprotokolliert, transkribiert und ins Deutsche übersetzt.

Im Rahmen der formulierenden Interpretation wurden die für das Erkenntnisinteresse relevanten Interviewpassagen einer thematischen Feingliederung in Ober- und Unterthemen unterzogen. Hierbei ging es um die Beantwortung der Frage nach dem «Was» der Beobachtung und der Erfassung des sogenannten immanenten Sinngehalts. Im Mittelpunkt der darauf aufbauenden reflektierenden Interpretation stand der (Orientierungs-) Rahmen, in welchem das Thema abgehandelt wird. Auf dieser Interpretationsebene kann das atheoretische Wissen der Interviewten expliziert werden. Die Standortgebundenheit des 
Forschers unterlag dabei einer «methodische[n] Kontrolle» (Bohnsack, 2008), das heißt, von Anbeginn wurde die Interpretation innerhalb der Forschergruppe intersubjektiv überprüft, um Motivunterstellungen zu vermeiden. Die Forschergruppe umfasste drei der Autorinnen sowie einen weiteren Kollegen. Ein dritter Analyseschritt gehörte der sinngenetischen Typenbildung mit einer umfassenden komparativen Analyse, die sich im fallübergreifenden Vergleich der Interviews vollzieht und nach Gemeinsamkeiten bzw. Kontrasten zwischen den Fällen sucht. Für die berichteten Ergebnisse im vorliegenden Artikel sind vor allem die beiden ersten Analyseschritte relevant.

\section{Quantitative Studie}

In der quantitativen Querschnittsstudie wurden zwischen Juni und Oktober 2013 insgesamt 194 ältere türkeistämmige MigrantInnen im Alter von 59 Jahren und mehr mittels persönlicher standardisierter Interviews befragt. Die Altersgrenze markiert in etwa den Eintritt in die (Vor-)Ruhstandsphase und die beginnende Herausbildung einer gesellschaftlich normierten Altersidentität. Da ältere türkeistämmige MigrantInnen, wie im vorherigen Abschnitt bereits belegt, eine verhältnismäßig schwer zugängliche Zielgruppe für wissenschaftliche Untersuchungen mit vergleichsweise geringen Beteiligungsraten darstellen, erfolgte die Stichprobenauswahl durch einen kombinierten Ansatz aus dem bewährten Netzwerkverfahren und einem Schneeballverfahren. VertreterInnen von Institutionen (z.B. Senioren-, Freizeit- und Nachbarschaftstreffs, Kultur- und Moscheevereinen, Pflegeeinrichtungen) sowie die bilingualen InterviewerInnen fungierten als Vertrauenspersonen. Mit ihrer Unterstützung wurden BefragungsteilnehmerInnen gewonnen, über die weitere InterviewpartnerInnen akquiriert werden konnten. Zusätzlich luden die InterviewerInnen potenzielle TeilnehmerInnen über Direktansprachen im öffentlichen Raum (z.B. in Herrencafés, Parks) ein, womit einer Stichprobenverzerrung zugunsten typischerweise in Institutionen vertretenen Personen entgegengewirkt werden sollte. Die Datenerhebung konzentrierte sich auf die Berliner Bezirke FriedrichshainKreuzberg, Mitte, Neukölln und Tempelhof-Schöneberg, die relativ hohe Anteile älterer türkeistämmiger EinwohnerInnen aufweisen, sich aber hinsichtlich der sozioökonomischen Lage, gemessen am Sozialindex 1 aus dem Sozialstrukturatlas 2008 (Senatsverwaltung für Gesundheit, Umwelt und Verbraucherschutz, 2008), unterscheidet. Die Zusammensetzung der Stichprobe wurde anhand der Merkmale Geschlecht, Alter und Bildungsstand kontrolliert. Hinsichtlich des Geschlechts wurde ein ausgeglichenes Verhältnis angestrebt und realisiert, um bei der relativ kleinen Stichprobengröße geschlechtsspezifische Analysen zu ermöglichen. Als Quote für das Merkmal Alter dienten Daten aus dem Berliner Einwohnermelderegister (Statistikamt Berlin-Brandenburg, 2013). Der höchste Bildungsabschluss wurde mittels Daten aus einer Sonderauswertung des Mikrozensus 2002 von Özcan und Seifert (2006) quotiert. Die Stichprobe folgte somit keiner Zufallsauswahl, bildet aber hinsichtlich der soziodemografischen Merkmale Alter und Bildungsstand annähernd die realen Verteilungen der türkeistämmigen Bevölkerung in Berlin ab.

Der Fragebogen wurde in deutscher Sprache erstellt, ins Türkische übersetzt und nach Durchführung eines Pretests und daraus folgender Anpassungen zur Prüfung der Übersetzungsqualität ins Deutsche zurück übersetzt. Da eine schriftliche Einwilligungserklärung als eine zusätzliche Teilnahmehürde für ältere türkeistämmige MigrantInnen eingestuft wurde, wurde die mündliche Einwilligung zur Teilnahme nach persönlicher und schriftlicher Aufklärung über den Hintergrund der Studie sowie ihren datenschutzrechtlichen Regelungen als informierte Zustimmung gewertet. Positive Voten eines behördlichen Datenschutzbeauftragten sowie einer Ethikkommission bzgl. dieses Vorgehens liegen vor.

Der Fragebogen erfasste u.a. Angaben zur Pendelmigration (wie viel Zeit im Jahr die Befragten in der Türkei verbringen) und zur Rückkehrorientierung (ob die Befragten dauerhaft in die Türkei ziehen möchten), zu Vorsorgeaktivitäten für das hohe Alter (mit welchen Möglichkeiten der Vorsorge für das hohe Alter die Befragten sich auseinandergesetzt haben) sowie zu den Pflegeerwartungen (durch wen die Befragten gepflegt werden möchten und ob eine migrationssensible Pflege für sie bedeutsam ist). Die Bedeutsamkeit von Migrationssensibilität in der Pflege wurde über sieben dichotome Einzelindikatoren abgefragt, welche Ergebnisse der qualitativen Studie sowie Empfehlungen für eine migrantenorientierte Pflegepraxis operationalisieren (vgl. z.B. Arbeiterwohlfahrt \& Caritasverband, 2006; von Bose \& Terpstra, 2012): die Versorgung durch türkischsprachiges Pflegepersonal, die Gewährleistung gleichgeschlechtlicher Pflege, die Berücksichtigung von Ernährungsgewohnheiten, von religiösen Feiertagen, das Ausziehen von Straßenschuhen bei Betreten der Wohnung, sowie die Erwartung, dass sich die Pflegekräfte mit der zu betreuenden Person auch unterhalten. Migrationssensible Pflege berücksichtigt mit dem Zuwanderungsstatus und der ethnischen Herkunft einhergehende Unterschiede im Vergleich zur autochthonen Mehrheitsbevölkerung. Diese Unterschiede können sich in kulturell geprägten und migrationsbedingten sozialen Praktiken manifestieren und in gleichförmigen, repetitiven und routinisierten Orientierungsmustern und Erwartungen an eine Pflege äußern. Das Vorliegen typischer Gemeinsamkeiten schließt dabei Individualität nicht aus. Erkenntnistheoretisch gesprochen findet sich vielmehr das Typische, das Allgemeine im Einzelnen, das Abstrakte im Konkreten (vgl. mit dieser Auffassung auch Kirchermeier, Uzarewicz, Klein \& Kutschke, 2003; Moebius, 2012). Diesem Umstand wird mit der Operationalisierung von Migrationssensibilität durch ein Item Rechnung getragen, das die Bedeutsamkeit des Eingehens auf individuelle Bedürfnisse und Wünsche erfasst. 
Die statistische Auswertung erfolgte mittels SPSS Statistics 22 und umfasst deskriptive, univariate Analysen sowie eine multiple Regression. Anhand der sieben Indikatoren zu Aspekten einer migrantenorientierten Pflege wurde ein Summenindex generiert, welcher die Valenz (Bedeutsamkeit) von Migrationssensibilität in der Pflege aus Nutzerperspektive abbildet. Dieser wurde in Beziehung zu potenziellen Einflussgrößen (Prädiktoren) gesetzt. Neben den Einflussgrößen «ethnische Identität» (deutsch / deutschtürkisch/türkisch) und «selbst eingeschätzte Deutschkenntnisse» ((sehr) gut/mittelmäßig/(sehr) schlecht) wurden das Alter sowie das Geschlecht als Kontrollvariablen in die Modellbildung mit einbezogen. Da die Normalverteilungsannahme der gebildeten Skala zur Valenz von Migrationssensibilität nicht erfüllt wurde, basiert die durchgeführte multiple Regression auf einem nonparametrischen Verfahren (Bootstrap). Zusätzlich zu den berücksichtigten Prädiktoren und Kontrollvariablen wurden die Einflussgrößen Rückkehrorientierung, Pendelmigration und subjektive Gesundheit sowie die Kontrollvariablen Pflegestufe und sozialer Status auf ihre Effekte hin kontrolliert. Da deren Koeffizienten nicht signifikant verschieden von Null sind, wurden diese Eingangsgrößen aus den Modellen entfernt. Wie aus Tabelle 5 hervorgeht, erklären die einbezogenen Regressoren insgesamt $12 \%$ der Varianz der Valenz von Migrationssensibilität aus Perspektive der MigrantInnen $(\mathrm{F}=4,93 ; \mathrm{p}<0,001)$.

Tabelle 2. Zusammensetzung der Stichprobe der qualitativen Studie

\begin{tabular}{ll}
\hline Alter & Anzahl \\
Arithmetisches Mittel & 67,9 Jahre \\
\hline Geschlecht & \\
Männlich & 6 \\
Weiblich & 5 \\
\hline
\end{tabular}

Höchster Berufsabschluss

Un- oder angelernt $\quad 5$

Ausbildung, Lehre, Meister/Techniker $\quad 4$

Hochschule, Universität

2

Zuwanderungsweg

Gastarbeiterzuwanderung (bis 1973)

11

Familienstand

Verheiratet

6

Verwitwet/geschieden/ledig

5

Religionszugehörigkeit

Islamisch

10

Alevitisch

1

Aufenthaltsdauer

Arithmetisches Mittel

42,2 Jahre

\section{Ergebnisse}

\section{Stichprobenbeschreibung}

Die Altersspanne der in der qualitativen Studie interviewten Türkeistämmigen der ersten Einwanderergeneration bewegte sich zwischen 60 und 79 Jahren. Einzelne InterviewpartnerInnen werden in der laufenden Ergebnisdarstellung kurz vorgestellt. Einen Gesamtüberblick über die soziodemografische Zusammensetzung des Samples gibt Tabelle 2 .

Die Zusammensetzung der Stichprobe der quantitativen Studie wird anhand der soziodemografischen Merkmale Alter, Geschlecht und Bildungsstand, mittels migrations- und integrationsbezogener Merkmale sowie dem Vorliegen einer Pflegestufe als pflegebezogenes Merkmal dargestellt (vgl. Tabelle 3).

Die 194 TeilnehmerInnen der quantitativen Studie waren zwischen 59 und 88 Jahre alt. Der Großteil (45\%) gehörte zur Gruppe der 65- bis 74-Jährigen, gefolgt von den 59- bis 64-Jährigen (38\%), den 75- bis 84-Jährigen (15\%) sowie den 85-Jährigen und Älteren (2\%). Hinsichtlich des Merkmals Geschlecht wurde ein etwa ausgeglichenes Verhältnis erzielt: Gut die Hälfte der Befragten (52\%) waren männlich und $48 \%$ weiblich. Die große Mehrheit der Befragten verfügt über ein eher geringes Bildungsniveau, gemessen am höchsten beruflichen Abschluss: $81 \%$ sind un- oder angelernt (niedriger Berufsabschluss), $12 \%$ schlossen eine Ausbildung, Lehre oder Meister ab (mittlerer Berufsabschluss) und nur $7 \%$ verfügen über einen Hochschul- oder Universitätsabschluss (hoher Berufsabschluss).

Die Mehrheit der BefragungsteilnehmerInnen bilden Zuwanderer der ersten Gastarbeitergeneration: 76 \% wanderten vor dem Anwerbestopp 1973, das heißt im Rahmen der Gastarbeiteranwerbung zu. Die restlichen $24 \%$ kamen erst später und somit über einen anderen Zuwanderungsweg wie dem Familiennachzug oder der humanitären Zuwanderung nach Deutschland. Mit Blick auf die Staatsangehörigkeit, die (subjektiven) Deutschkenntnisse und die (subjektive) ethnische Identität kennzeichnet die Befragten ein eher geringer Integrationsstand. Nur ein Viertel (25\%) der Befragten verfügt über die deutsche Staatsangehörigkeit, weitaus mehr (68\%) über die Türkische und $6 \%$ über eine doppelte Staatsbürgerschaft. Gut ein Drittel der Befragten (35\%) schätzt seine Deutschkenntnisse als gering und fast die Hälfte (47\%) als mittelmäßig ein. Nur $18 \%$ der Interviewten gaben an, über gute Deutschkenntnisse zu verfügen. Dementsprechend fühlen sich $62 \%$ der Befragten als Türken, $32 \%$ als Deutsch-Türken und nur $6 \%$ als Deutsche.

Gemessen an der Pflegestufe bezieht der Großteil der Interviewten keine Leistungen der Pflegeversicherung; $76 \%$ gaben an, in keine Pflegestufe eingruppiert zu sein, $20 \%$ verfügen über eine Pflegestufe und $4 \%$ wussten nicht, ob sie eine Pflegestufe haben bzw. machten hierzu keine Angabe. 
Tabelle 3. Zusammensetzung der Stichprobe der quantitativen Studie

\begin{tabular}{lcc}
\hline \multicolumn{1}{l}{ Alter } & Anzahl & Prozent \\
59-64 Jahre & 74 & 38 \\
65 - 74 Jahre & 87 & 45 \\
$75-84$ & 29 & 15 \\
85 und älter & 3 & 2 \\
Gesamt & 193 & 100 \\
\hline
\end{tabular}

\section{Geschlecht}

\begin{tabular}{crr} 
Männlich & 100 & 52 \\
Weiblich & 94 & 48 \\
Gesamt & 194 & 100 \\
\hline
\end{tabular}

\begin{tabular}{lrr}
\hline Höchster Berufsabschluss & & \\
Un- oder angelernt & 158 & 81 \\
Ausbildung, Lehre, Meister/Techniker & 23 & 12 \\
Hochschule, Universität & 13 & 7 \\
Gesamt & 194 & 100
\end{tabular}

Zuwanderungsweg

Gastarbeiterzuwanderung (bis 1973) $\quad 146 \quad 76$

Anderer Zuwanderungsweg (ab 1974) $\quad 45 \quad 24$

Gesamt

$191 \quad 100$

Staatsangehörigkeit

\begin{tabular}{lrr} 
Türkische & 133 & 68 \\
Deutsche & 48 & 25 \\
Doppelte & 11 & 6 \\
Andere/Keine & 2 & 1 \\
samt & 194 & 100 \\
\hline
\end{tabular}

Deutschkenntnisse

$\begin{array}{lrr}\text { Gut } & 34 & 18 \\ \text { Mittelmäßig } & 91 & 47 \\ \text { Gering } & 67 & 35 \\ \text { Gesamt } & 192 & 100\end{array}$

Ethnische Identität

$\begin{array}{lrr}\text { Deutsch } & 11 & 6 \\ \text { Türkisch } & 110 & 62 \\ \text { Deutsch-Türkisch } & 57 & 32 \\ \text { Gesamt } & 178 & 100\end{array}$

\section{Pflegestufe}

\begin{tabular}{lrr} 
Ja & 37 & 20 \\
Nein & 143 & 76 \\
Keine Angabe/weiß nicht & 7 & 4 \\
Gesamt & 187 & 100 \\
\hline
\end{tabular}

Quelle: Schenk, Krobisch \& Ikiz, 2014.

Anmerkung. Die Gesamtzahl der Befragungsteilnehmer variiert je nach berichtetem Merkmal aufgrund fehlender Werte.

\section{Befragungsergebnisse}

Ein Blick auf die Rückkehrorientierungen und Pflegeerwartungen der Befragten liefert zunächst Anhaltspunkte, inwieweit davon auszugehen ist, dass ältere türkeistämmige MigrantInnen als Akteure multikultureller Pflegearrangements auftreten. Daran anschließend werden Pflegeerwartungen betrachtet, die Rückschlüsse auf die Bedeutung des gemeinsamen Migrations- bzw. soziokulturellen Hintergrundes mit den Pflegenden und einer migrationssensiblen Pflege zulassen.

\section{Geringe Rückkehrorientierung - Altern in Deutschland}

Inwieweit möchten ältere Türkeistämmige ihren Lebensabend in Deutschland verbringen? Bekanntermaßen charakterisiert die sogenannte Gastarbeitergeneration eine starke Rückkehrorientierung. Die häufig vertagte Rückkehrentscheidung stellt sich für ältere MigrantInnen bei Eintritt in das Rentenalter mit neuer Nachdrücklichkeit und beinhaltet die Entscheidung darüber, wo und wie sie bei Bedarf gepflegt werden möchten. Für die pflegerische Versorgung in Deutschland ist mit der Rückkehrentscheidung die Frage verknüpft, welche Bedeutung älteren Türkeistämmigen als Nutzergruppe in der Pflege zukommt. Laut Befragungsergebnissen der quantitativen Studie sieht die Mehrheit der TeilnehmerInnen ihren Alterswohnsitz in Deutschland. Von den 193 Befragten, die diese Frage beantworteten, möchten $63 \%$ in Deutschland bleiben. Ein knappes Viertel (23\%) hegt weiterhin die Absicht, in die Türkei zurückzukehren; für $13 \%$ kommt eine Rückkehr vielleicht in Frage. Dass ältere Türkeistämmige eher in Deutschland als in der Türkei Pflege in Anspruch nehmen werden, darauf verweist auch der geringe Anteil (17\%) der 185 Befragten, der sich schon einmal mit einer Rückkehr als Möglichkeit der Vorsorge für das hohe Alter auseinandergesetzt hat. Dies spricht dafür, dass ältere Türkeistämmige eine zunehmend relevante Gruppe (potenziell) Pflegebedürftiger in Deutschland bilden.

\section{Wachsende Offenheit für professionelle Pflege}

Inwieweit ist mit älteren Türkeistämmigen als NutzerInnen professioneller Pflege zu rechnen? Befragt nach ihren Pflegeerwartungen wird ersichtlich, dass der familialen Pflege nach wie vor eine große Bedeutung in der türkeistämmigen Bevölkerung zukommt. Insbesondere der/die EhepartnerIn wird in der Pflicht gesehen. Von insgesamt 188 Personen, welche die Frage nach den Pflegeerwartungen an ihre Mitmenschen beantworteten, stimmten $77 \%$ $\mathrm{zu}$, dass der/die EhepartnerIn die Pflege älterer Menschen übernehmen sollte. Geringere Erwartungen richten die Befragten an die Kinder mit einer Präferenz für eine Pflege 
durch die Töchter (55\%) gegenüber den Söhnen (47\%). Trotz der Relevanz familialer Pflege deuten die Befragungsdaten auch auf eine große Offenheit für eine Versorgung durch professionelle Pflegepersonen hin; $89 \%$ der Befragten ist der Meinung, ältere Menschen sollten (auch) professionell gepflegt werden.

Die Ergebnisse der qualitativen Interviews förderten eine entsprechende Hierarchie der Pflegeerwartungen zutage.

I: Nun ja, wenn Sie selber pflegebedürftig werden?

A: Da sollen andere dran denken (lacht) (..) Was soll ich machen ich, \{was\} Gott nicht gebe, wenn du nun so was wie gelähmt bist, was wirst du tun? (.) Dann gibt es gar nichts, was ich machen kann. (A 329-332)

Ahmet (m) wurde im Jahr 1947 geboren, ist 1973 als Gastarbeiter nach Deutschland zugewandert und Vater von drei Kindern. Er spielt zwar mit dem Gedanken, als Rentner in die Türkei zurückzukehren oder zwischen Deutschland und der Türkei zu pendeln. Dennoch dokumentieren sich in dem Zitat typische Orientierungen der türkeistämmigen Befragten. Die Imagination von Pflegebedürftigkeit verknüpft Ahmet mit einem absoluten Verlust von Selbstständigkeit und der Annahme, komplett auf Hilfe angewiesen zu sein. Diesen Zustand symbolisiert der Begriff Lähmung. Insbesondere seine Frau sieht er hier in der Pflicht, ihn zu pflegen oder für pflegerische Unterstützung zu sorgen.

I: Aber es gibt Leute, die was für Sie tun können.

A: Klar Liebes, gibt es wird es geben, daran soll die Frau denken. (A 333-334)

Hasan (m), im Jahr 1945 geboren und 1974 als Gastarbeiter nach Deutschland migriert, Vater von fünf Kindern, ist voller Zuversicht, im Falle eines Pflegeeintritts Unterstützung zu erhalten.

I: Nun gut, wenn du selbst eines Tages auf Hilfe angewiesen sein solltest, hast du daran gedacht, was sein wird?

$H$ : Wenn ich selber auf Pflege angewiesen sein sollte, natürlich sagen wir dann, wir für uns selber, viel gibt es den Gedanken nicht. Warum, weil ich sage, wir haben eine Familie. In dem Gedanken wir lassen jemanden uns pflegen.

I: Was heißt jemand, zum Beispiel wer?

H: Das kann unbedingt jemand von außerhalb sein oder nun //hmh// die Tochter kümmert sich, wenn wir das sagen, jeder hat seine Wohnung. Also dies es findet sich jemand, der Materielles braucht oder aber, wir sagten ja, Altersheim, was weiß ich, so einen Gedanken hatte ich nicht ... (H 648-659)

Zunächst rechnet Hasan mit der Pflegebereitschaft seiner Familienmitglieder, daher sorgt er sich nicht um einen möglichen Pflegeeintritt. Eine gewisse Unsicherheit und Ambivalenz bezüglich der Pflegebereitschaft seiner Familienmitglieder dokumentieren sich dann doch in dem Hinweis, Pflegeleistungen auch von außerhalb in Anspruch zu nehmen. Explizit erwähnt Hasan aber wiederum seine Tochter als nahe Familienangehörige, die ihn pflegen könnte. Hasan betont hierbei, dass Tochter und Eltern in je eigenen Wohnungen leben. Vermutlich stellt er in Rechnung, dass seine Tochter mit eigener Familie die Pflege des Vaters nur schwer tragen könnte. Dies mag auch den sich anschließenden Ver- weis auf eine bezahlte Pflegekraft oder auf ein Altersheim erklären. Sofern familiale Pflege thematisiert wird, schreiben sowohl Ahmet als auch Hasan vornehmlich den weiblichen Familienmitgliedern die Pflegerolle zu.

Bei einem Pflegeeintritt erwarteten die Interviewten also zuvorderst Unterstützung von den EhepartnerInnen, dann von den Kindern, insbesondere den (Schwieger-)Töchtern. Falls die nahen Angehörigen des potenziell Pflegebedürftigen nicht imstande sein sollten, die Pflege zu übernehmen, wird als nächstes auf fernere Verwandte gezählt. Erst wenn im familialen Rahmen eine Pflege nicht realisiert werden kann, wird eine professionelle bzw. institutionell organisierte Pflege in Betracht gezogen. Demnach könnte die in der quantitativen Studie ermittelte Offenheit für die Versorgung durch professionelle Pflegepersonen auf ein bestehendes Bewusstsein und die Akzeptanz eines Rückgangs des familialen Unterstützungspotenzials und dem damit einhergehenden Erfordernis alternativer Versorgungsmodelle verweisen. Dafür spricht auch, dass $29 \%$ der Befragten der quantitativen Studie die Töchter und 34 \% die Söhne von jeglicher Pflegeverantwortung freisprechen.

\section{Bedeutung kulturell- und migrationsbedingter Erwartungen an eine Pflege}

Ob und inwiefern messen türkeistämmige Ältere kulturellund migrationsbedingten Aspekten in der Pflege Bedeutung bei? Als essenziell erwies sich vor allem die gemeinsame Sprache als Voraussetzung für eine erfolgreiche Verständigung und den Austausch mit den Pflegefachpersonen. Türkischkenntnisse der Pflegepersonen gehören für die große Mehrheit (79\%) der TeilnehmerInnen der quantitativen Studie zu einer guten professionellen Pflege. Der Sprache wird - so das Ergebnis der qualitativen Studie - eine instrumentelle und eine emotionale Funktion zugewiesen.

Leyla (w), Jahrgang 1936 geboren und 1974 als Gastarbeiterin nach Deutschland gekommen, wurde aufgrund eines Arbeitsunfalls in die Frührente entlassen und konnte nach den Operationen nicht mehr alleine wohnen. Seither lebt sie in einer türkischen Pflegeeinrichtung. In den folgenden Zitaten begründet Leyla diese Wahl.

L: Danach wollten sie mich in ein deutsches Heim schicken, aber ich bin nicht gegangen. Ich sagte: «Mein Deutsch ist nämlich nicht ausreichend. Nicht, dass sie mich \{nicht\} gut versorgen werden, aber ich kann mich nicht gut verständlich machen.»Ich sagte: «Es gibt türkische Ruhehäuser, da gehe \{ich\} hin.» Und so bin ich im letzten Jahr hierher gekommen. Das heißt ein Jahr ist um. (.) Ich kam am füften des siebten Monats. //I: Mhm////Mhm// (K3w1: 286-291) L: Warum ich nicht ein Deutsches Heim wollte. Weil ich nicht imstande bin, meinen Kummer und meine Angelegenheiten zum Ausdruck zu bringen. Deshalb wollte ich nicht. (K3w1: 76-78)

Für Leyla scheint es eine Selbstverständlichkeit, unabhängig vom kulturellen Hintergrund eine gute Pflege zu erhalten. Dennoch war die Sorge, sich nicht verständlich 
machen zu können, der entscheidende Auslöser für die Wahl eines muttersprachlichen Pflegeangebots. Der Begriff Kummer verweist darauf, dass es dabei nicht nur um die Verständigung auf der Sachebene («Angelegenheiten») geht. Vielmehr besitzt - neben der instrumentellen Funktion der Sprache - auch ein Austausch in seelischen Belangen einen hohen Stellenwert.

Auch Hasan betont die emotionale Komponente der Muttersprache.

I: Nun ja, //hmh// du hast wohl die Pflegeheime erwähnt. Pflege, es gibt türkische Pflegeheime zum Beispiel hier. // $\mathrm{hmh} / /$ Was denkst du dazu?

H: Zu ihnen [gedehnt] meine Ansicht natürlich wir nun // hmh// da wir Türken sind, die Sprache ist hier sehr wichtig. Um unsere Sorgen, Nöte zu sagen eventuell //hmh// sie behandeln uns noch emotionaler, positiver, mäßiger daher empfinde ich Freude. Dass solche Plätze von Türken eröffnet werden. (K7m1: 290-296)

H: Die Liebe und Sympathie dort [in der Türkei] ist noch anders. (K7m1: 584-585)

Darüber hinaus dokumentiert sich in dieser Interviewpassage, dass mit der Pflege durch Landsleute eine größere Nähe antizipiert wird. Gemeinsamkeiten in tradierter Geschichte und Kultur sowie ein geteilter aktueller Erlebniszusammenhang erleichtern ein Verstehen im Sinne eines unmittelbaren intuitiven Verstehens. In dem beschriebenen Sinne ist die gemeinsame Sprache auch die Voraussetzung für eine soziale Einbindung, die sich viele Befragte von der Versorgungsbeziehung wünschen. So geht für $83 \%$ der Befragten eine gute Pflege über die bloßen körper- und haushaltsbezogenen Leistungen hinaus; sie möchten sich mit den Pflegefachpersonen auch unterhalten können. Neben der gemeinsamen Sprache scheinen für die Teilnehmer der quantitativen Studie kulturelle Aspekte vor allem bei haushalts- und körperbezogenen Tätigkeiten wichtig zu sein. Nahezu einig waren sich die Befragten, dass eine «gute» Pflegefachperson den Wohnraum nicht mit Straßenschuhen betritt (89\%) und ihre Essgewohnheiten berücksichtigt werden (87\%). Knapp drei Viertel (72\%) der Befragten möchte, dass die Körperpflege von Pflegefachpersonen des gleichen Geschlechts erbracht wird. Weniger bedeutend erwiesen sich indes religiöse Aspekte, wobei auch die Mehrheit der Befragten religiöse Feiertage von den Pflegepersonen beachtet wissen möchte. Mit 59 \% fällt dieser Anteil jedoch deutlich geringer aus als bei den anderen Facetten guter professioneller Pflege. Kulturell- und migrationsbedingte Aspekte, und insbesondere die Sprache, sind aus Sicht der Befragten von Bedeutung. Trotz der Gemeinsamkeiten macht für die Befragten auch eine Individualpflege eine gute professionelle Pflege aus; fast alle (92\%) legen Wert darauf, dass eine Pflegefachperson auf ihre individuellen Wünsche und Bedürfnisse eingeht. Ab-

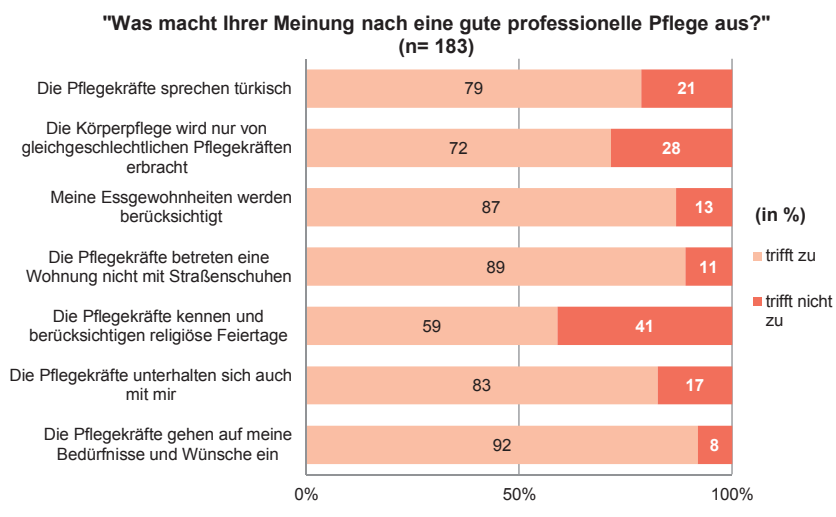

Abbildung 1. Kulturell- und migrationsbedingte Erwartungen an eine Pflege in \% (Quelle: Schenk, Krobisch \& Ikiz, 2014).

bildung 1 zeigt die quantitativen Ergebnisse zu den kulturell- und migrationsbedingten Erwartungen an eine Pflege im Überblick.

Welche Einflussgrößen üben nun einen Effekt darauf aus, ob befragte MigrantInnen mehr oder weniger viele kulturell- und migrationsbedingte Aspekte als bedeutsam in der Pflege erachten? Tabelle 4 beschreibt die Zielvariable «Valenz der Migrationssensibilität in der Pflege». Je höher der Indexwert, desto größer die Bedeutsamkeit von migrationssensiblen Aspekten in der Pflege. Das arithmetische Mittel von 5,59 zeigt, dass ein relativ hoher Anteil der Befragten dazu tendiert, eine Vielzahl von migrationssensiblen Merkmalen als relevant zu erachten.

Die in die Regression eingehenden Einflussgrößen «ethnische Identität» sowie «Deutschkenntnisse» erweisen sich als signifikante Prädiktoren (vgl. Tabelle 5). Da es sich hierbei um Dummy-Variablen handelt, gibt der unstandardisierte Koeffizient die Abweichung zur Referenzkategorie «Deutsche Identität» bzw. «(sehr) gute Deutschkenntnisse» an. Die geschätzten standardisierten Regressionskoeffizienten können hingegen als Indikator der Effektstärke der Prädiktoren interpretiert werden. Den stärksten signifikanten Effekt auf die subjektive Valenz von migrationssensibler Pflege übt die gefühlte ethnische Identität der BefragungsteilnehmerInnen aus $(\beta=0,36, p<0,05)$. Personen mit einer türkisch geprägten Identität weisen einen um 1,16 höheren Summenindex-Wert auf, als jene BefragungsteilnehmerInnen, die sich als Deutsche verstehen. Das heißt, sie wählten etwa einen Indikator mehr aus, der ihrer Meinung nach für eine gute professionelle respektive migrationssensible Pflege relevant ist. Gefühlte Deutsch-Türken zeigen einen ähnlichen Effekt, dieser ist allerdings nicht signifikant. Einen signifikanten Einfluss auf die Wertigkeit migrationssensibler Pflegeaspekte für (potenziell) pflegebedürftige türkeistämmige MigrantInnen weist hingegen der Grad an Deutschkenntnissen auf. Die Bedeutsamkeit einer migrationssensiblen Pflege ist sowohl bei

Tabelle 4. Abhängige Variable «Valenz Migrationssensibilität» (gebildet über Summenindex)

\begin{tabular}{lcccc}
\hline Variable & Minimum & Mittel & Median & Maximum \\
\hline Migrationssensibilität & 1 & 5,59 & 6 & 7 \\
\hline
\end{tabular}


Tabelle 5. Multiple Regression zur Vorhersage der Valenz von Migrationssensibilität

\begin{tabular}{|c|c|c|c|}
\hline & Unstandardisierte Koeffizienten & Standardfehler & Standardisierte Koeffizienten \\
\hline Prädiktoren & $B$ & SE & $\beta$ \\
\hline \multicolumn{4}{|l|}{ Ethnische Identität } \\
\hline Deutsch-Türkisch & 0,91 & 0,60 & 0,27 \\
\hline Türkisch & 1,16 & 0,56 & $0,36 *$ \\
\hline \multicolumn{4}{|l|}{ Deutschkenntnisse } \\
\hline Mittel & 1,03 & 0,31 & $0,33 * \star \star$ \\
\hline Schlecht & 0,77 & 0,34 & $0,23 *$ \\
\hline \multicolumn{4}{|l|}{ Kovariate } \\
\hline Alter & $-0,004$ & 0,01 & $-0,01$ \\
\hline Geschlecht (Mann) & $-0,73$ & 0,25 & $-0,23 * \star$ \\
\hline Konstante & 4,48 & 1,37 & $-0,01 * * \star$ \\
\hline$N$ & 165 & & \\
\hline $\mathrm{R}^{2}$ & 0,15 & & \\
\hline Korr. $\mathrm{R}^{2}$ & 0,12 & & \\
\hline
\end{tabular}

Die Koeffizienten beruhen auf nicht-parametrischen Bootstrap auf 5000 Bootstrap-Stichproben; $\beta$ basieren zusätzlich auf z-standarisierten $B-W e r t e n$. Abhängige Variable: Valenz von Migrationssensibilität

${ }^{* * *} p<0,001 ;{ }^{* *} p<0,01,{ }^{*} p<0,05$

Personen mit mittleren $(\beta=0,33, \mathrm{p}<0,001)$ sowie mit schlechten $(\beta=0,23, p<0,05)$ Deutschkenntnissen gegenüber MigrantInnen mit gut ausgebildeten deutschen Sprachkenntnissen erhöht. Ein im Vergleich zu den Prädiktoren signifikanter Effekt auf die Wertigkeit von Migrationssensibilität zeigt sich zudem für die Kontrollvariable Geschlecht. Männliche Befragungsteilnehmer wählten um ca. 0,73 weniger Indikatoren einer migrationssensiblen Pflege aus als die weiblichen Befragten $(\beta=-0,23, \mathrm{p}<0,01)$.

Deutschkenntnisse und die ethnische Identität nehmen einen zentralen Einfluss darauf, wie ausgeprägt kulturellund migrationsbedingte Erwartungen an eine Pflege bei älteren Türkeistämmigen sind.

\section{Diskussion}

Die Förderung der Beschäftigung von Menschen mit Migrationshintergrund sowie die Zunahme Pflegebedürftiger mit Migrationshintergrund führen $\mathrm{zu}$ einer ethnischen und kulturellen Pluralisierung in der professionellen Pflege in Deutschland. Kaum Aufmerksamkeit erhielt bisher die Frage, wie tragfähig die damit einhergehenden multikulturellen Pflegearrangements sind. Während erste Forschungsergebnisse zu den Dynamiken und Effekten multikultureller Teams in der Pflege vorliegen (vgl. Stagge, 2016), nimmt der vorliegende Beitrag den Aspekt der Versorgungsbeziehungen von Pflegefachpersonen und Pflegeempfängern mit unterschiedlicher soziokultureller Prägung in den Blick. Untersucht wurde, welche Akzeptanz solche Versorgungsbeziehungen möglicherweise bei (potenziellen) türkeistämmigen NutzerInnen finden. Dies wird anhand der Frage diskutiert, welche Relevanz ein gemeinsamer Migrations- bzw. soziokultureller Hintergrund in der Beziehung zwischen Pflegebedürftigen und Pflegefachpersonen für ältere Türkeistämmige besitzt.

Die Studienergebnisse weisen zunächst darauf hin, dass ältere Türkeistämmige eine relevante (potenzielle) Nutzergruppe multikultureller Pflegearrangements darstellen. Zwar hegt immerhin ein Viertel der Befragten den Wunsch, ihren Lebensabend in der Türkei zu verbringen, jedoch bleibt ungewiss, wie handlungsrelevant diese Orientierung ist. Die deutliche Mehrheit möchte in Deutschland bleiben. Gestützt wird die Einschätzung, dass ein Großteil der älteren Türkeistämmigen in Deutschland alt werden möchte, durch andere Studienergebnisse. Danach hegen beispielsweise ältere türkeistämmige PendelmigrantInnen zwar die Idealvorstellung von einer familialen altersbedingten Pflege im Herkunftsland (vgl. Strumpen, 2012). Gleichzeitig besteht jedoch die Präferenz, den Lebensmittelpunkt im Krankheits- und Pflegefall nach Deutschland zu verlagern. Ausschlaggebend hierfür scheinen einerseits der Wunsch, den Kontakt zu der in Deutschland verwurzelten zweiten und dritten Generation aufrecht zu erhalten, und andererseits die vergleichsweise größere soziale und gesundheitliche Sicherheit in Deutschland (vgl. Krumme, 2004; Prätor, 2009; Strumpen, 2012; Wettich, 2007). Zudem zeigen die Ergebnisse der multiplen Regression, dass Rückkehrorientierung und Pendelmigration in keinem signifikanten Zusammenhang mit der Wertigkeit migrationssensibler Pflegeaspekte für (potenziell) pflegebedürftige ältere türkeistämmige MigrantInnen stehen. 
In der großen Offenheit für professionelle Pflege und den eher geringen Pflegeerwartungen an die Kinder zeichnet sich möglicherweise ein wachsendes Bewusstsein dafür ab, dass das familiale Unterstützungspotenzial abnimmt und eine professionelle Versorgung als «letzte Instanz» damit unausweichlich wird. Dies deckt sich mit bestehenden Studienergebnissen, wonach familiale Pflege weiterhin als Idealvorstellung verbreitet ist und professionelle Pflege erst denkbar wird, wenn keine Angehörigen für die Pflege zur Verfügung stehen (vgl. Strumpen, 2012; Kohls, 2012). Auch eine wachsende Offenheit für professionelle Pflege wurde bereits antizipiert und darauf zurückgeführt, dass die Kinder älterer Türkeistämmiger eine Pflege nicht leisten können oder wollen und Einstellungen zu professioneller Pflege pragmatischer würden (vgl. Prätor, 2009; Seibel-Erdt \& Şöhret, 1999; Zimmermann, 2012). Demnach ist davon auszugehen, dass ältere Türkeistämmige als NutzerInnen professioneller Pflege auftreten werden - insbesondere zur Ergänzung familialer Pflege.

Die Tragfähigkeit von Versorgungsbeziehungen in multikulturellen Pflegearrangements wird vor dem Hintergrund der vorgestellten Befragungsergebnisse kritisch zu diskutieren sein. Für einen Großteil der älteren Türkeistämmigen erscheint eine Versorgung durch Pflegefachpersonen mit einem anderen Migrations- bzw. soziokulturellen Hintergrund als der eigene nur bedingt geeignet, zumindest aber nicht mit einem Teil ihrer Erwartungen zu harmonieren. Denn neben dem Wissen um und der Beachtung von kulturellen und religiösen Gepflogen- und Gewohnheiten vor allem im körper- und haushaltsbezogenen Bereich stellt aus Nutzerperspektive insbesondere eine Versorgung durch Landsleute eine zentrale Qualität professioneller Pflege dar. Entscheidend ist die Möglichkeit zur sachlichen und kulturell geprägten intuitiven Kommunikation in der Muttersprache. Dabei geht es zum einen um einen effektiven Austausch zu pflegebezogenen Fragen. Mindestens ebenso wichtig scheint aber auch der Aspekt der sozialen Einbindung zu sein. Letztere kann nach Ansicht der Befragten nur in der Versorgungsbeziehung mit Pflegefachpersonen mit einem ähnlichen soziokulturellen Hintergrund erzielt werden, da mit ihnen eine größere emotionale Nähe und Vertrautheit möglich ist. Dies ist insbesondere dann der Fall, wenn die eigenen Deutschkenntnisse als eher gering eingeschätzt werden und sich die Befragten eher der türkischen als der deutschen Kultur zugehörig fühlen und entsprechend die eigene ethnische Identität bestimmen. Sprachliche Verständigung als ein Qualitätskriterium in der Pflege thematisierte auch eine der Studien zur CareMigration (van Holten, Jähnke \& Bischofberger, 2013). Darüber hinaus messen türkeistämmige Frauen einer migrationssensiblen Pflege signifikant häufiger als Männer Bedeutung bei. Unter welchen Voraussetzungen multikulturelle Pflegekonstellationen bei älteren türkeistämmigen MigrantInnen auf Akzeptanz stoßen, sollte Gegenstand weiterer Forschung sein. Die Befragungsergebnisse weisen auf große Gemeinsamkeiten bei den kulturell- und migrationsbedingten Pflegeerwartungen älterer türkeistämmiger MigrantInnen hin. Zugleich findet die Auffassung, dass eine gute Pflege auch individuelle Wünsche und Bedürfnisse berücksichtigt, große
Zustimmung durch die Befragten. Migrationssensible Pflege sollte demnach auch Individualpflege beinhalten.

Die hier vorgestellten Ergebnisse beziehen sich auf die heute ältere türkeistämmige Bevölkerung, eine Zuwanderergruppe, die über eher geringe Deutsch- und andere Fremdsprachenkenntnisse verfügt und die häufig eine starke lebensweltliche Orientierung an der eigenen ethnischen Community aufweist (vgl. Haug, 2008; Okken et al., 2008). Erste belastbare Anhaltspunkte zu dieser Frage liefern neuere Untersuchungsergebnisse. Danach misst die erste Generation Türkeistämmiger einem ähnlichen kulturellen Hintergrund der Pflegekraft - und somit auch einer Versorgung in der Muttersprache - größere Bedeutung bei als ihre Nachkommen (Schultz \& Wittlif, 2015). Gleiches gilt für die Übertragbarkeit der Ergebnisse auf andere Gruppen älterer MigrantInnen. Eigene Forschungsergebnisse deuten aber auch hier darauf hin, dass derselbe soziokulturelle Hintergrund von Pflegepersonen eine Rolle spielt. Beispielsweise dann, wenn dadurch eine quasifamiliale Pflegesituation hergestellt wird und so tradierte Pflegeorientierungen beispielsweise unter russlanddeutschen MigrantInnen nicht beschädigt werden (Schenk et al., 2011).

Auch für die ältere Bevölkerung ohne Migrationshintergrund liegt der Schluss nahe, dass soziokulturelle Gemeinsamkeiten tragfähige Versorgungsbeziehung begünstigen. So weisen Ergebnisse anderer Studien auf die große Relevanz guter Deutschkenntnisse von Pflegekräften (Schulz \& Wittlif, 2015) und verbreitete Vorurteile und fremdenfeindliche Einstellungen in dieser Gruppe hin (Zick, Küpper \& Hövermann, 2011). Soziokulturelle Differenzen könnten demnach die Beziehung zwischen Pflegeempfängern und Pflegefachpersonal beeinträchtigen. Folglich ist anzunehmen, dass multikulturelle Pflegekonstellationen auch bei der Versorgung der deutschen Bevölkerung ohne Migrationshintergrund mit Herausforderungen einhergehen.

Aufgrund der regionalen Beschränkung beider Studien auf den Stadtstaat Berlin können die Ergebnisse nicht ohne weiteres auf die ältere türkeistämmige Bevölkerung in Deutschland insgesamt übertragen werden. Die Stichprobe repräsentiert jedoch annähernd die Verteilung der Merkmale Alter und Bildung in der älteren türkeistämmigen Bevölkerung in Berlin, was für eine Gültigkeit der Ergebnisse auf regionaler Ebene spricht. Wünschenswert wäre eine bevölkerungsrepräsentative Studie, welche die Ergebnisse validiert und auch in Relation zu anderen Migrantengruppen sowie der Bevölkerung ohne Migrationshintergrund setzt. Trotz der Einschränkungen liefern die Daten erste wichtige Ergebnisse zu den Pflegeerwartungen einer zunehmend relevanten Gruppe und zur Bedeutung des gemeinsamen Migrations- bzw. soziokulturellen Hintergrunds in Versorgungsbeziehungen.

\section{Schlussfolgerungen}

Der Migrations- bzw. soziokulturelle Hintergrund von Pflegefachpersonen spielt bei der Pflege älterer Türkei- 
stämmiger der ersten Generation eine zentrale Rolle. Den hier vorgestellten Ergebnissen zufolge legt diese Gruppe großen Wert auf eine Versorgung durch Personen, die einen ähnlichen biografischen und soziokulturellen Erlebniszusammenhang erfahren haben wie sie selbst. Der gemeinsam geteilte Kommunikations- und Handlungsrahmen bildet für sie eine wichtige Voraussetzung für eine tragfähige Versorgungsbeziehung und gute Pflege. Da viele ältere Türkeistämmige ihren Lebensabend voraussichtlich in Deutschland verbringen und dort zunehmend als NutzerInnen professioneller Pflege auftreten werden, steht die pflegerische Versorgung vor der Herausforderung, im Spannungsfeld zwischen den Anforderungen an eine Pflege und der zunehmenden ethnischen und kulturellen Pluralisierung unter den Pflegefachpersonen eine angemessene Versorgung $\mathrm{zu}$ organisieren. Eine verstärkte Ausbildung und Beschäftigung von türkeistämmigen Pflegefachpersonen erscheint hier ein möglicher Lösungsansatz. Mit Blick auf den Fachkräftemangel in der Pflege werden jedoch auch multikulturelle Pflegekonstellationen unumgänglich sein. Diese gilt es, migrationssensibel zu gestalten, damit die kulturell geprägten Anforderungen Berücksichtigung finden.

\section{Förderung}

Empirische Grundlage des Beitrags bilden zwei von der Stiftung «Zentrum für Qualität in der Pflege〉 (ZQP) geförderten bzw. beauftragten Studien (Schenk, Krobisch \& Ikiz, 2014; Schenk, Meyer, Maier, Aronson \& Gül, 2011).

\section{Beiträge der einzelnen Autorinnen}

Substanzieller Beitrag zu Konzeption oder Design der Arbeit: LS, KG, PA (qual. Studie); VK, LS (quant. Studie) Substanzieller Beitrag zur Erfassung, Analyse oder Interpretation der Daten: LS, KG, PA (qual. Studie); VK, PTS, LS (quan. Studie)

Manuskripterstellung: VK, LS, PTS, KG

Einschlägige kritische Überarbeitung des Manuskripts: VK, LS, PTS

Genehmigung der letzten Version des Manuskripts: VK, PTS, KG, PA, LS

Übernahme der Verantwortung für das gesamte Manuskript: VK, LS

\section{Literatur}

Afentakis, A.; Maier, T. (2010). Projektionen des Personalbedarfs und -angebots in Pflegeberufen bis 2025. Statistisches Bundesamt, Wirtschaft und Statistik 11,990-1002.
Arbeiterwohlfahrt Kreisverband Friedrichshain-Kreuzberg e.V.; Caritasverband für das Erzbistum Berlin e.V.. (2006). Ältere Migrantinnen und Migranten in Berlin - Eine Handreichung. www. kompetenzzentrum-altenhilfe.de/pdf/handreichung1.pdf.

Bermejo, I.; Hölzel, L.-P.; Kriston, L.; Härter M. (2012). Subjektiv erlebte Barrieren von Personen mit Migrationshintergrund bei der Inanspruchnahme von Gesundheitsmaßnahmen. Bundesgesundheitsblatt, 55 (8), $944-953$.

Bohnsack, R. (2008). Rekonstruktive Sozialforschung - Einführung in qualitative Methoden. 7. Aufl. Opladen/Farmington Hills: Barbara Budrich, UTB.

Borde, T. (2005). Repräsentation ethnischer Minderheiten in Studien und Gesundheitsberichten. Erfordernis, Chancen und Nebenwirkungen. In: Borde, T.; David, M.; (Hrsg.), Kinder und Jugendliche mit Migrationshintergrund. Lebenswelten, Gesundheit und Krankheit. Frankfurt am Main: Mabuse, $267-287$.

Bose, A.; Terpstra, J. (2012). Muslimische Patienten pflegen. Praxisbuch für Betreuung und Kommunikation. Berlin/Heidelberg: Springer.

Braeseke, G.; Merda, M.; Bauer, T.; Otten, S.; Stroka, M.; Talmann, A. (2013). Migration - Chancen für die Gewinnung von Fachkräften in der Pflegewirtschaft. Bundesgesundheitsblatt, 56,1119-1126.

Bundesministerium für Gesundheit (2015). Pflegefachkräftemangel. www.bmg.bund.de/themen/pflege/pflegekraefte/pflegefachkraeftemangel.html [05.03.2016].

Dietzel-Papakyriakou, M.; Olbermann, E. (2005). Gesundheitliche Lage und Versorgung alter Arbeitsmigranten in Deutschland. In: Marschalck, P.; Wiedl, K. (Hrsg.), Migration und Krankheit. Göttingen: V\&R unipress.

Haug, S. (2008). Sprachliche Integration von Migranten in Deutschland. Working Paper 14. Nürnberg: Bundesamt für Migration und Flüchtlinge.

Kelle, U.; Kluge, S. (1999). Vom Einzelfall zum Typus. Fallvergleich und Fallkontrastierung in der qualitativen Sozialforschung. Opladen: Leske und Budrich.

Kirchermeier, S.; Uzarewicz, C.; Klein, H.; Kutschke, T. (2003). Kollektivistische und individualistische Dimensionen in den Grundlagen kultursensibler Pflege und Gesundheitsförderung. Pflege \& Gesellschaft, 8 (1), 3 - 10.

Kohls, M. (2011). Morbidität und Mortalität von Migranten in Deutschland. Forschungsbericht 9. Nürnberg: Bundesamt für Migration und Flüchtlinge.

Kohls, M. (2012). Pflegebedürftigkeit und Nachfrage nach Pflegeleistungen von Migrantinnen und Migranten im demographischen Wandel. Forschungsbericht 12. Nürnberg: Bundesamt für Migration und Flüchtlinge.

Krumme, H. (2004). Fortwährende Remigration: Das transnationale Pendeln türkischer Arbeitsmigrantinnen und Arbeitsmigranten im Ruhestand. Zeitschrift für Soziologie, 33 (2), 138 - 153.

Moebius, S. (2012). Kulturforschungen der Gegenwart - die Studies. In: Moebius, S. (Hrsg.), Kultur. Von den Cultural Studies bis zu den Visual Studies. Eine Einführung. Bielefeld: transcript: 7-12.

Okken, P.; Spallek, J.; Razum, O. (2008). Pflege türkischer Migranten. In: Bauer U.; Büscher A. (Hrsg.). Soziale Ungleichheit und Pflege. Beiträge sozialwissenschaftlich orientierter Pflegeforschung. Wiesbaden: VS Verlag für Sozialwissenschaften, 396- 422.

Özcan, V.; Seifert; W. (2006). Lebenslagen älterer Migrantinnen und Migranten in Deutschland. Deutsches Zentrum für Altersfragen (Hrsg.), Lebenssituation und Gesundheit älterer Migranten in Deutschland: Expertisen zum Fünften Altenbericht der Bundesregierung, Bd. 6, 7-75.

Prätor, S. (2009). Alter und Altersbilder in der Türkei und bei türkischen Immigranten. In: Ehmer, J.; Höffe, O. (Hrsg.), Bilder des Alters im Wandel. Nova Acta Leopoldina N. F., 99 (363).

Razum, O.; Zeeb, H. (1998). Epidemiologische Studien unter ausländischen Staatsbürgern in Deutschland - Notwendigkeit und Beschränkungen. Das Gesundheitswesen, 60, 283 - 286.

Reiss, K.; Dragano, N.; Ellert, U.; Fricke, J.; Greiser, K.-H.; Keil, T. et al. (2014). Comparing sampling strategies to recruit migrants for an epidemiological study. Results from a German feasibility study. The European Journal of Public Health, 1 - 6. 
Sass, A.; Grüne, B.; Brettschneider, A.-K.; Rommel, A., Razum, O., Ellert, U. (2015). Beteiligung von Menschen mit Migrationshintergrund an Gesundheitssurveys des Robert Koch-Instituts. Bundesgesundheitsblatt, 58, 533-542.

Schenk, L. (2002). Migrantenspezifische Teilnahmebarrieren und Zugangsmöglichkeiten im Kinder- und Jugendgesundheitssurvey. Gesundheitswesen, 64, Sonderheft 1,59-68.

Schenk, L. (2008). Gesundheit und Krankheit älterer und alter Migranten. In: Kuhlmey, A.; Schaeffer, D. (Hrsg.), Alter, Gesundheit und Krankheit. Handbuch Gesundheitswissenschaften. Bern: Hans Huber.

Schenk, L.; Meyer, R.; Maier, A.-S.; Aronson, P.; Gül, K. (2011). Rekonstruktion der Vorstellungen vom Alter und von Einstellungen zur (stationären) Pflege bei Personen mit Migrationshintergrund Bericht für das ZQP. Berlin: Zentrum für Qualität in der Pflege. www.zqp.de/upload/content.000/id00015/attachment01.pdf [04.2014].

Schenk, L.; Krobisch, V.; Ikiz, D. (2014). Pflegesituation von türkeistämmigen älteren Migranten und Migrantinnen in Berlin - Bericht für das ZQP. Berlin: Zentrum für Qualität in der Pflege. www.zqp.de/upload/content.000/id00015/attachment03.pdf [11.2014].

Schimany, P.; Rühl, S.; Kohls, M. (2012). Ältere Migrantinnen und Migranten. Entwicklungen, Lebenslagen, Perspektiven. Forschungsbericht 18. Nürnberg: Bundesamt für Migration und Flüchtlinge.

Schmidt, M.; Schneekloth, U. (2011). Abschlussbericht zur Studie «Wirkungen des Pflege-Weiterentwicklungsgesetzes». Bericht zu den Repräsentativerhebungen im Auftrag des Bundesministeriums für Gesundheit. Berlin: Bundesministerium für $\mathrm{Ge}-$ sundheit.

Schultz, C.; Wittlif, A. (2015). In Vielfalt altern. Pflege und Pflegepräferenzen im Einwanderungsland Deutschland. Policy Brief des SVR-Forschungsbereichs 2015-2. Berlin: Sachverständigenrat deutscher Stiftungen für Integration und Migration (SVR).

Seibel-Erdt, R.; Şöhret, A. (1999). Nicht ganz hier und nicht mehr zu Hause: Gespräche mit Türkinnen und Türken der ersten Generation. Münster: Waxmann.

Senatsverwaltung für Gesundheit, Umwelt und Verbraucherschutz (2008). Sozialstrukturatlas 2008. Gesundheitsberichterstattung Berlin. www.berlin.de [08.04.2014].

Stagge, M. (2016). Multikulturelle Teams in der Altenpflege. Eine qualitative Studie. Wiesbaden: Springer VS.

Statistikamt Berlin-Brandenburg (2013). Statistisches Informationssystem Berlin-Brandenburg (StatIS-BBB). www.statistikberlin-brandenburg.de/datenbank/inhalt-datenbank.asp [08.04.2014].

Strumpen, S. (2012). Altern in fortwährender Migration bei älteren Türkeistämmigen. In: Baykara-Krumme H.; Motel-Klingebiel, A.; Schimany P. (Hrsg.), Viele Welten des Alterns. Ältere Migranten im alternden Deutschland. Wiesbaden: Springer VS, 411- 433.

Ulusoy, N.; Gräßel, E. (2010). Türkische Migranten in Deutschland. Wissens- und Versorgungsdefizite im Bereich häuslicher Pflege - ein Überblick. Zeitschrift für Gerontologie und Geriatrie 43 (5), $330-338$. van Holten, K.; Jähnke, A.; Bischofberger, I. (2013). Care-Migration - transnationale Sorgearrangements im Privathaushalt (Obsan Bericht 57). Neuchâtel: Schweizerisches Gesundheitsobservatorium.

Wengler, A. (2013). Ungleiche Gesundheit. Zur Situation türkischer Migranten in Deutschland. Frankfurt am Main: Campus.

Wettich, J. (2007). Migration und Alter: Kulturelle Altersbilder im Wandel. Saarbrücken:VDM Verlag Dr. Müller.

Yilmaz, Y.; Glodny, S.; Razum, O. (2009). Soziale Netzwerkarbeit als alternatives Konzept für die Rekrutierung türkischer Migranten zu wissenschaftlichen Studien am Beispiel des saba Projekts. Hallesche Beiträge zu den Gesundheits- und Pflegewissenschaften, 8 (48).

Zimmermann, H.-P. (2012). Altersbilder von türkischen Migrantinnen und Migranten in Deutschland im Vergleich. Islamische Grundsätze - alltägliche Sichtweisen. In: Baykara-Krumme H.; Motel-Klingebiel, A.; Schimany P. (Hrsg.), Viele Welten des Alterns. Ältere Migranten im alternden Deutschland. Wiesbaden: Springer VS, 315-337.

Zick, A.; Küpper, B.; Hövermann, A. (2011). Die Abwertung der Anderen. Eine europäische Zustandsbeschreibung zu Intoleranz, Vorurteilen und Diskriminierung. Berlin: Friedrich-Ebert-Stiftung.

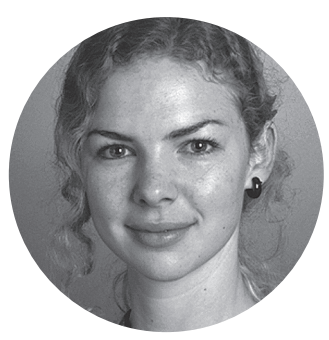

\section{Verena Krobisch}

Institut für Medizinische Soziologie und Rehabilitationswissenschaft Charité - Universitätsmedizin Berlin Luisenstraße 57

10117 Berlin

verena.krobisch@charite.de

\section{Was war die größte Herausforderung bei Ihrer Studie?}

Die Vertrauensbildung auf Seiten der (potenziellen) BefragungsteilnehmerInnen, die unserer Studie häufig skeptisch gegenüber standen.

\section{Was wünschen Sie sich bezüglich der Thematik für die Zukunft?} Vergleichende Studien zu den Pflegeorientierungen Älterer mit und ohne Migrationshintergrund.

\section{Was empfehlen Sie den LeserInnen zum Weiterlesen/Vertiefen?}

Einen weiteren wichtigen Aspekt ethnischer und kultureller Pluralisierung in der Pflege beleuchtet Maya Stagge in ihrer Forschungsarbeit zu «Multikulturellen Teams in der Altenpflege» (siehe Literaturverzeichnis).

Manuskripteingang: 17.11.2015

Manuskript angenommen: 03.06.2016 